\title{
Making it personal - population health management and the NHS
}

\author{
Martin McShane \\ Optum, London, UK, and \\ Karen Kirkham \\ Department of Primary Care, NHSE/I and Dorset Clinical Commissioning Group, \\ Dorset, $U K$
}

\begin{abstract}
Purpose - Changes in demographics and disease patterns are challenging health and care systems across the world. In England, national policies have reset the direction of travel for the NHS. Collaboration, integration and personalisation are intended to become prime principles and drivers for new models of care. Central to this is the concept of population health management. This has emerged, internationally, as a method to improve population health. Fundamental for population health management to succeed is the use of integrated data, analytics combined with professional insight and the adoption of a learning health system culture. This agenda reaches beyond the NHS in England and the public health profession to embrace a broad range of stakeholders. By drawing on international experience and early experience of implementation in the United Kingdom, the potential for health and care systems in England to become world leading in population health management is explored. Design/methodology/approach - A viewpoint paper.

Findings - Population health management is a major change in the way health and care systems look at the challenges they are facing. It makes what is happening to individuals, across the continuum of care, the essence for insight and action. The NHS has the components for success and the potential to become world leading in delivery of population health management as part of its integrated care agenda.

Originality/value - This is the first viewpoint paper to set out how population health management contributes to the integrated care agenda in the NHS.
\end{abstract}

Keywords Population health management, Population health, NHS

Paper type Viewpoint

\section{Introduction}

The NHS Long Term Plan (NHS, 2019) makes extensive references to population health management (PHM). We want to explore and illustrate what we believe this means, based on international evidence, practical experience of supporting implementation in the United States, coupled with understanding, development and early implementation in the NHS drawing on Dorset's experience of participating in the NHS PHM development programme.

\section{What is population health management?}

PHM has emerged in recent decades as a methodology that uses data to guide the planning and delivery of care to achieve maximum impact on population health (Buck et al., 2018). NHS

(C) Martin McShane and Karen Kirkham. Published by Emerald Publishing Limited. This article is published under the Creative Commons Attribution (CC BY 4.0) licence. Anyone may reproduce, distribute, translate and create derivative works of this article (for both commercial \& non-commercial purposes), subject to full attribution to the original publication and authors. The full terms of this licence may be seen at http://creativecommons.org/licences/by/4.0/legalcode

Dedicated to the memory of Dr Craig Wakeham who was a GP for 30 years in Dorset, pioneered integrated nursing teams and was Chief Clinical Information Officer. He sadly died from Covid-19 in April 2020.

The Population Health Management Development Programme was funded and commissioned by NHSE/I. The authors would like to thank the support provided by the NHSE/I Population Health Management team, Optum UK, Dorset Clinical Commissioning Group and local providers.

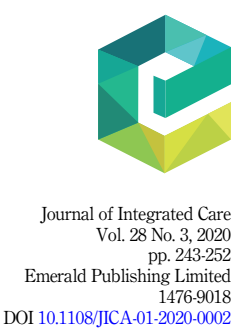


JICA

28,3

244

England provides the following definition: Population health management (PHM) - is helping us understand our current, and predict our future, health and care needs so we can take action in tailoring better care and support with individuals, design more joined up and sustainable health and care services and make better use of public resources.

It is how we use historical and current data to understand what factors are driving poor outcomes in different population groups. It is how we then design new proactive models of care which will improve health and well-being at present as well as in 20 years' time. This could be by stopping people becoming unwell in the first place, or, where this isn't possible, improving the way the system works together to support them. (PHM, 2019).

Central to PHM is the ability to link data for individuals and apply analytic tools which, coupled with professional knowledge and experience of the population and individuals being served, changes attitudes and behaviours to care delivery right across the continuum of health and care. The nature, purpose and impact of using such data are discussed further.

\section{Population health analytics}

In the United Kingdom, the health and care system is well positioned to create new insights for professionals and individuals by linking and analysing multiple sources of data. Starting with NHS data from general practice, hospital, community and mental health services, a holistic picture based on biological and psychological factors can be created. As confidence and trust grow, such data can be linked to social care, other social data (e.g. housing, employment) and third-sector data sources. In the future, individuals could also contribute data from wearables and apps. Analysing this linked data set using analytics such as actuarial analysis, segmentation and risk stratification with algorithms and machine learning reframes how the system of care is perceived from one traditionally focussed on organisations and conditions to one that is based on the experience of individuals, the complexity they manage and the risk they face. Sub-segmenting the population based on complexity and demographics has proved helpful in engaging professionals in understanding where to look and where to start with PHM. There are various ways to segment a population such as Bridges to Health (Lynn et al., 2007) or the National Association of Primary Care approach (NAPC, 2019). Optum has used the Symmetry tool (Symmetry Episode Risk Groups, 2018) to support segmentation and risk stratification adapted to local approaches to segmentation where necessary. These techniques help those who fund the system and providers of care anticipate needs, intervene proactively and seek to consistently address risk factors in a way which uses resources to best effect supporting better experience and outcomes for individuals and enabling clinicians and other professionals to organise care effectively, proactively and efficiently.

\section{Changing behaviours}

While the linked data and analysis are essential, the real importance is in how it is used to influence the behaviour of professionals delivering care and individuals managing their own lives; how it is used to support a holistic, team-based, bio-psycho-social approach to care. This change in perspective and how data needs to be linked and used underpins a number of fundamental changes the NHS will have to undergo and support, with other stakeholders, if it is to realise the potential of PHM. As we, and others, have found the data is essential but is greatly enhanced with the knowledge and experience frontline professionals bring to the interpretation and application of that data (Ross et al., 2017).

\section{Transforming the operating model}

The need to transform the operating model for health and care is being driven by enormous changes in the challenges health and care systems face worldwide (Boyd and Fortin, 2010). These challenges are due to past successes. People are living longer, due to factors including 
but also beyond healthcare advances. Treatments and technology mean that many conditions, once fatal, are now "chronic". This has altered the pattern of disease, deficit and distress in the population. Yet, fundamentally, the health and care system would be recognisable to someone from the mid-20th century. It remains predominantly reactive, fragmented, biomedically focussed, with the individual as a passive recipient of the benevolence of the system. It is accepted that this needs to change and all parts of the world are exploring how this can be done with many common themes emerging (Britnell, 2015a).
Population health management and the NHS

\section{Policy drivers}

The NHS Long Term Plan envisions the change for the NHS and is accompanied by two, perhaps equally, important policy documents in Universal Personalised Care (2019) and investment and evolution: a five-year framework for GP contract reform to implement. GP contract: Investment and Evolution (2019). Universal Personalised Care seeks to give individuals more choice and control over their health and care through implementing six components.

(1) Shared decision-making

(2) Personalised care and support planning

(3) Enabling choice, including legal rights to choice

(4) Social prescribing and community-based support

(5) Supported self-management

(6) Personal health budgets and integrated personal budgets

The new GP contract for investment and evolution is designed to revitalise and reposition primary care as a key driver for PHM through primary care networks (PCNs) as they are intended to be "an essential building block of every Integrated Care System".

\section{Effecting change}

Learning from practical, tactical implementation of accountable care in the United States, Optum has distilled essential capabilities to effectively support the transition from a reactive, fragmented, condition and organisationally focussed system to one which makes the individual central and continuity of care possible in line with Haggerty's principles of informational, management and relational continuity. As pointed out "most patients assume that transfer of information is seamless and that care is coordinated, so that gaps in care arrive as bad surprises. This shakes their trust and confidence in professions... and potentially to become non-adherent to treatment" (Haggerty, 2012). Addressing such issues through PHM aligns with the intent of the Long Term Plan and enabling policies.

As set out earlier, an integrated data set is used to segment and stratify the population (deploying algorithms and other techniques) to support prioritising interventions to improve the way resources are used and improve quality. Informed by the data, designed by professionals and those with lived experience, chosen interventions can then be used to model mitigated scenarios to support future financial and workforce planning.

Vitally the next step in the process requires the right information governance so that professionals with appropriate permissions can re-identify individuals who will benefit. In essence, the process builds insight from individuals' experiences to create a population view. This only becomes purposeful when it can be reconnected as actionable insight for individuals connecting with professionals. It requires a cycle of continuous improvement that drives intent from person to system and system to person: a learning health system (http:// www.learninghealthcareproject.org/section/background/learning-healthcare-system). 
JICA

28,3

246

This approach has received positive feedback from participants in the PHM development programme as the principles of a learning health system connect with the motivation of professionals. It also embraces and accepts the complexity of the system, as discussed further.

\section{A framework for delivery}

Delivery is facilitated by appropriate provider networks. As Gawande points out, modern health care is a team game crossing both professional and organisational boundaries (Gawande, 2010).

Information technology supports integrated working but only with the right system governance and incentives aligned in support. Change on this scale requires investment in organisational (or system) development. Scaling and sustaining the change desired is not easy (Kumpunen et al., 2019). PHM needs systems, organisations, teams, professionals to "think big but start small" and embrace methodologies that acknowledge the complexity of such change if it is to be scaled and sustained (see Figure 1). This includes:

(1) Acknowledging unpredictability,

(2) Recognising self-organisation,

(3) Facilitating interdependencies,

(4) Encouraging sense-making,

(5) Developing adaptive capability in staff,

(6) Attending to human relationships,

(7) Harnessing conflict productively

(Greenhalgh and Papoutsi, 2019).

Through supporting the development of numerous accountable care organisations in the United States, Optum has found a framework (Figure 2) invaluable in delivering the change in culture, systems and processes required. The flipside is that no single capability will effect the change required. Systems need to ensure multiple components are supported for successful integrated care.

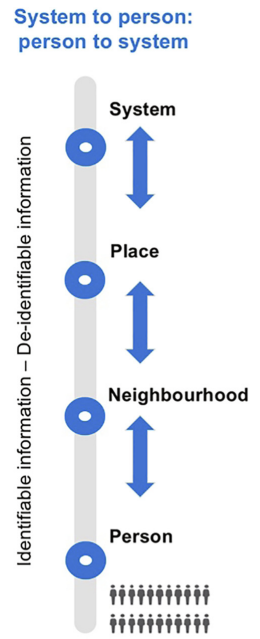

Analytics provided in programme

Economic modelling \& actuarial projections to look at changes in population health and care needs and how to mitigate health and financial risk across care settings

Costed segmentation to identify high and rising risk cohorts. Benchmarking and variation across providers and population segments. Predictive modelling on interventions and Return on Investment (ROI)

Drill down into segments through risk stratification and impactability modelling to support proactive case finding. Addressing variation by segment

Improving population health by enabling integrated teams at every level to make data-driven decision
Patient level theographs to support care redesign and personalised care, and analyse individual care pathways

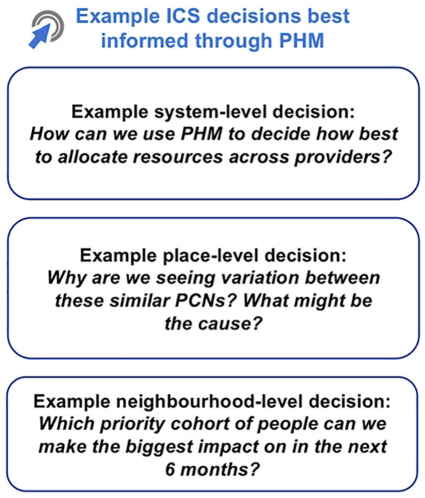

Example person-level decision: How can I leverage our collective assets to help this person who is at risk? 


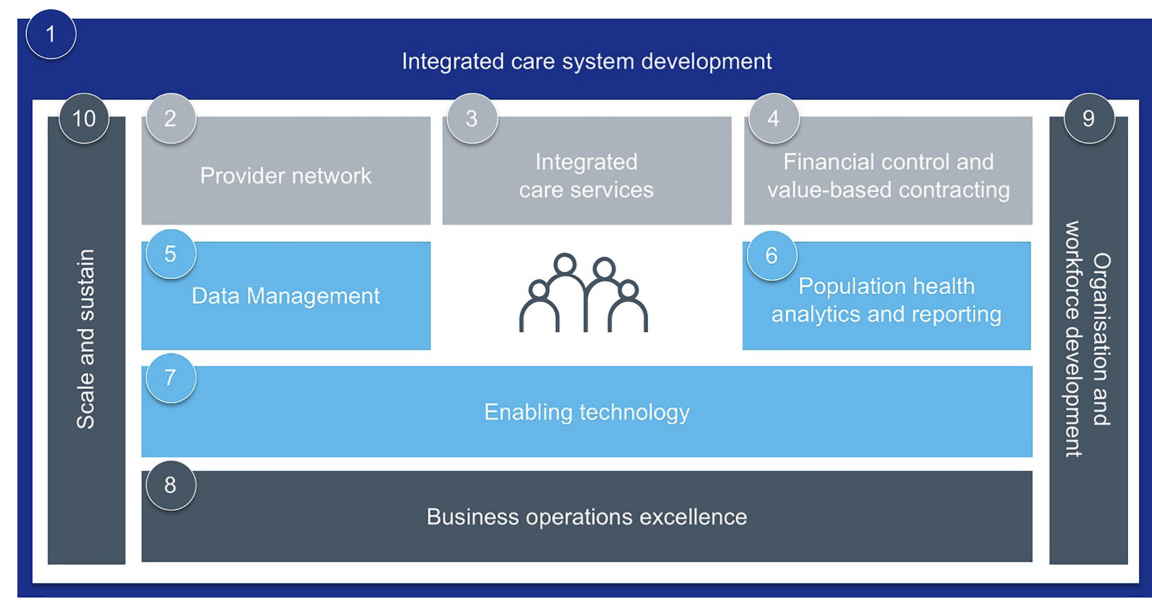

Population health management and the NHS

\section{NHS relevance}

Applying this approach in the NHS in England since the Long Term Plan was announced has been encouraging. In many ways the NHS and wider system are perfectly poised to accelerate adoption of integrated care and go above and beyond systems which are currently considered more advanced. The most significant advantages are that it has a single unique identifier (the NHS number) for users of services and, in effect, a single payer (government funding). It also has (although under considerable strain) strong foundations for primary care at scale. The importance and impact of a high-quality primary care have been evidenced for decades (Allen et al., 2018) and a prime example of how it can drive PHM is Clalit in Israel which produces good health outcomes based on a longstanding commitment to community and family practice medicine with a strong public health orientation. (Britnell, 2015b).

\section{Learning how to implement PHM in the NHS}

In 2019, four nascent integrated care systems in the NHS participated in a programme to advance their development and understanding of PHM.

NHS England supported by Optum worked with the four systems over an intensive 20-week programme to take what integrated data was available and work with emerging PCNs to implement change in the management of a locally determined cohort of patients.

\section{Learning how to enable PHM in the NHS}

Dorset was one of the first wave of integrated care systems to undertake the programme.

\section{The data and development of insights}

The question for those of us leading in these new integrated care systems is to understand how to turn intelligence into insights and then into action. Development and implementation of a PHM programme essentially require a fresh and integrated approach to take the data to clinical teams at a local level to facilitate intelligent decision-making. It requires analysts to work in a different way with clinical and management teams. In time this will almost certainly lead to a rethinking of the multi-professional team approach needed to deliver care differently with potentially new types of contracting.

The programme cemented the importance of having analysts collaborating for the system versus individual organisations, so they could collectively provide insights for PHM. Dorset had not resourced a linked data set and the first step was to get this in place. 
JICA

28,3

248

With programme support, and sustained engagement with data controllers, the (not inconsiderable) challenges to create a linked data set were overcome and a local solution implemented over a period of weeks.

Despite taking longer than initially anticipated, the teams learnt how to solve their own problems with the support of Optum and NHSE/I and NHS Digital, Dorset formed its own PHM data platform; The Our Dorset intelligent working programme (IWP) was established to support the ambitions of the NHS Long Term Plan and PHM programme in Dorset as part of the Digitally Transformed Dorset STP portfolio, to improve the way information is used to support service improvement, planning and development.

IWP and the Dorset Intelligence and Insight Service (DiiS) will deliver, seek to support the infrastructure and intelligence strands of PHM, providing the technical infrastructure in the form of a linked data set (at individual level) with established information governance processes containing data from across our integrated care system (ICS) partners, intelligence analytics and the skilled analytical workforce to make best use of the rich data available to us.

This assists clinicians with risk stratification, population segmentation, audit, clinical decision support, service management, planning and commissioning. A shared data repository containing pseudonymised patient-level linked data from Dorset's health and social care organisations will provide a single source of information for joined up and consistent analysis. Data sets relating to "people" activity (including NHS patients, clients and service users in social care), finance, workforce, estates, quality and population, data currently held by local authorities such as town planning, schools and deprivation statistics. The data will be de-identified as appropriate and then shared appropriately for purposes such as population health analytics, service planning, training and research and risk stratification of people for direct care.

The programme collects data currently from approximately $80 \%$ of practices and is set to deliver an updated data stream every $24 \mathrm{~h}$ for the Dorset ICS by the end of the year.

Aligning the necessary information governance and data processing agreements and processes takes time and is dependent on trusted relationships between partners as well as technical ability. Dorset's practices (independent contractors as is the model in the NHS in England) had never before shared data in this way. Clear information governance guidance was developed for them to feel secure in participating in the programme - and a highly professional approach was needed to enable this to happen - undermining the necessity of strong and trusting relationships across the system. In addition, undertaking this process deepened the relationships between analysts and PCNs in Dorset, with analysts becoming part of the clinical and management teams, sitting together to find the answers the clinicians asked of the data - a powerful triad. This has led to development and training opportunities to promote the role of the analysts within the system leading to the development of a training academy and has enabled $\mathrm{PCNs}$ to develop the infrastructure required for a mature $\mathrm{PCN}$ ( ref Maturity Matrix NHSE/I 2019).

Developing and progressing the DiiS requires investment and needs to be prioritised along with other pressing clinical needs, and this remains a challenge to any system wishing to research and develop such an important tool.

\section{Developing primary care}

Alongside the development of the PHM programme, Dorset was transforming its general practice environment to create strong and resilient PCNs. There are now 18 PCNs in Dorset, each covering a population of around 30,000-76,000 patients across both an urban and a rural geography. Each PCN is aligned with local community boundaries, so serves a single local geography, with a strong community focus which lends itself to enquiry about the health of its local population, the variations in health and an opportunity to target gaps and unmet 
need as well as opportunity to work with colleagues across the voluntary sector, social care, housing and education. This provides the ideal infrastructure for a change using the PHM approach - which has largely, to date, not been successful with local public health initiatives perhaps because a truly integrated approach between health, care and public health partners has until now not flourished in part due to silos and fragmented thinking within systems. The new policy direction around integrated care and the development of PCN infrastructure provides the ideal foundation for this way of working and the potential is huge for real change to occur.

Armed and informed by the data, PCNs in Dorset were given autonomy to decide for themselves on which groups of patients they wished to focus. This is important because meaningful change is more likely to be sustained when local clinicians are invested and own that change - a bottom-up approach to transformation (Swanwick and McKimm, 2011).

The approach allowed PCNs to build on their existing priorities and ensured PHM was not seen as a separate exercise to their own development, but an essential part of their journey to maturity. Allowing PCNs to make these decisions themselves showed them the value of using data for their populations and promoting culture change in how data is used. This does, however, pose challenges. There is a learning need for clinicians and their staff to understand and then unlock the potential for quality improvement by reviewing and interrogating the data they possess and then translating insights into actions. This approach has not been one that has systematically been embedded in undergraduate or postgraduate education but must now be considered to form part of the curriculum for clinicians of many specialities as it is directly applicable to many environments. Interrogation of the data allowed each PCN to understand the risk profiles and segmentation of the local population and understand their own opportunities for improving care.

\section{A learning system}

The second challenge was about creating a learning environment with protected time for clinicians and managers in an already stretched general practice system. Key to success was planning well ahead and absolutely prioritising and supporting practices to release clinical and managerial time.

Then there is the culture of transformational change. Developing a new approach to the way we deliver care and asking groups of practices to come together to agree to a new way of working requires skill, leadership and considerable investment in time. But general practice is innovative and agile, and if we can harness these attributes and allow an organic and bottomup approach to change, then we must surely be able to realise the potential of this programme to unlock real improvements in care for local populations. Some areas have progressed at a faster pace than others, and clinical leadership has proved pivotal in determining the rate of change within local networks. A distributed leadership approach is likely to lead to sustained change.

National policy expressly asking for evidence of embedding QI approaches as part of PHM for GPs, for example, at appraisal could lead to a greater pace of change, as could investment nationally in this way of working.

\section{A system view}

System-level actuarial modelling is being progressed looking at the best possible value approach [https://www.futurefocusedfinance.nhs.uk/decision-framework-bpv]. Giving local economic and actuarial analysis to PCNs for their own population proved a powerful motivator for change and brought to light patients whose needs were not being best met but were quickly able to be looked after differently. Further segmentation and then risk profiling 
JICA

28,3

of specific cohorts of patients will give each network a specific and manageable number of patients for whom redesign of interventions could be planned, implemented and then analysed. This in turn will lead to more refined workforce planning.

Care models should be implemented to focus on early intervention, anticipatory care and proactive interventions to prevent illness, reduce hospitalisations and reduce inequalities and transform care from a reactive to a proactive model as is referenced in the Long Term Plan

Systems (ICS or STP) could choose to invest to drive this change with a long-term planning view.

\section{Person-centred care}

PCNs have developed a personalised approach to the changing care models with interventions that focus on understanding what matters to patients. As well as identifying people for specific interventions, PCNs used the approach as an opportunity to tailor and personalise care around individual patients. PCNs started to design care models that used a risk-stratified approach to care and designed new care models that gave people with complex needs more time and a multi-professional approach and sought to understand what their personal goals were to keep them healthy and happy. Some of the most powerful examples worked across the boundaries of primary, secondary and social care.

\section{Workforce}

PHM is now showing how we need to design our workforce differently in some areas, but also informing us that we must develop the workforce across traditional boundaries and increase multi-professional learning. This links closely to the evolution of new care models within the NHS, with new roles and responsibilities developing across traditional boundaries. The additional roles described in the new GP contract in England in 2020 support this approach and NHSE funding over the next five years will enable the implementation of new roles within multi-professional teams at PCN level. We need to ensure that sufficient workforce planning will occur to meet the needs of the local population, with great attention on multi-professional learning together.

\section{Evaluation}

Ongoing evaluation must be a core part of the transformation process. We must be prepared to halt schemes that are not delivering the change we want to see, quickly. We must also be ready to spread and scale new care models that are clearly beneficial, and the system around us needs to be able to align financial and workforce changes to support these new care models as they develop.

Dorset is now making PHM a core part of system and supporting PCN development, by scaling the approach and using a peer-to-peer learning environment.

Dorset's is freeing up analytical resource to dedicate to providing PHM insights for system leaders and PCNs, with the next wave of $\mathrm{PCNs}$ receiving similar support to that provided by the programme.

Dorset is developing a segmentation tool for its PCNs that can be used by clinicians to identify individual people to follow up with.

There are some key success factors that have emerged from the Dorset pilot.

(1) A strong consensus from the leadership within the ICS across that this was the correct course of action, and leaders supported the development programme and the ongoing roll out, but also enabled the right level of governance and decision-making to ensure success.

(2) Business intelligence and data-led approaches were fundamental to targeting care improvement and cost reduction programmes in Dorset's sustainability and 
transformation plan. Dorset had been a part of the successful Wessex bid for Local Health Care Record Exemplar (LHCRE) funding. A Dorset Care Record is being developed as a shared care record for frontline clinical staff. While neither of these could yet provide the data needed for PHM, it showed Dorset was fertile ground for developing a PHM approach. It also demonstrated a commitment to building the underlying infrastructure needed for PHM in the future.

(3) Momentum behind a linked data set. Dorset had already seen the value of linked data in one-off projects on chronic obstructive pulmonary disorder (COPD) and type 2 diabetes. These projects had focussed on linking data for a specific disease and did not link information across care pathways. There was a system-wide recognition that this approach would not encourage a holistic view of people and services. A linked data set is necessary to get a whole picture of the complexity patients and clinicians encounter every day. Dorset's belief that a holistic view of the data is needed motivated them to overcome issues that previously prevented them from linking data.

(4) A bottom-up approach to engaging staff clinical champions for PHM was identified prior to the start of the programme. This meant there was a ready-made group of GPs that saw the value in PHM and were ready to drive it forward. It also adds sustainability to the programme by spreading knowledge and expertise about PHM across the system. Some of these leaders are now our most vocal ambassadors for a PHM approach to improvement.

Dorset has undoubtedly been on a journey of discovery with PHM. Dedicated and talented clinicians and managers have worked tirelessly to solve some wicked problems within our system, addressing technical challenges and solving problems internally.

Dorset has valued the essential contribution of business analysts and clinical and support teams, providing support and time to build the infrastructure needed to implement a PHM approach.

We look forward to building on these strong foundations to improve quality of care for patients and reduce inequalities, provide the best experience for patients, ensure value for money and help bring the joy of working in committed teams to the workplace.

\section{Conclusion}

PHM is a major change in the way health and care systems look at the challenges they are facing. It makes what is happening to individuals, across the continuum of care, the essence for insight and action. Our view is that the NHS has the components for success and the potential to become world leading in delivery of PHM.

\section{References}

Allen, L.N., Barkley, S., De Maeseneer, J., van Weel, C., Kluge, H., de Wit, N. and Greenhalgh, T. (2018), "Unfulfilled potential of primary care in Europe", BMJ, Vol. 363, p. k4469, doi: 10.1136/ bmj.k4469.

Boyd, C.M. and Fortin, M. (2010), "Future of multimorbidity research: how should understanding of multimorbidity inform health system design?", Public Health Reviews, Vol. 32, No 2, pp. 451-474.

Britnell, M. (2015a), In Search of the Perfect Health System, Palgrave, London.

Britnell, M. (2015b), Israel. The Best Kept Secret in Global Health? In Search of the Perfect Health System, pp. 69-73, Palgrave, London.

Buck, D., Baylis, A., Durka, D. and Robertson, R. (2018), A Vision for Population Health, The King's Fund, available at: https:/www.kingsfund.org.uk/sites/default/files/2018-11/A\%20vision\% 20for\%20population \%20health\%20online\%20version.pdf (accessed 16 February 2020). 
Gawande, A. (2010), Cowboys and Pit Crews, the New Yorker, May 26th 2010, available at: https:// www.newyorker.com/news/news-desk/cowboys-and-pit-crews (accessed 14 November 2019).

Greenhalgh, T. and Papoutsi, C. (2019), "Spreading and scaling up innovation and improvement", $B M J$, Vol. 365, p. 12068, doi: 10.1136/bmj.12068.

Haggerty, J.L. (2012), "Ordering the chaos for patients with multimorbidity”, BMJ, Vol. 345, e5915, doi: 10.1136/bmj.e5915.

Kumpunen, S., Edwards, N., Georghiou, T. and Hughes, G. (2019), Evaluating Integrated Care: Why Are Evaluations Not Producing the Results We Expect? Briefing, Nuffield Trust, available at: https://www.nuffieldtrust.org.uk/resource/evaluating-integrated-care-why-are-evaluations-notproducing-the-results-we-expect (accessed 18 Feburay 2020).

Lynn, J., Straube, B.M., Bell, K.M., Fencks, S.F. and Kambic, R.T. (2007), "Using population health segmentation to provide better health care for all: the "Bridges to health", Model The Millbank Quarterly, Vol. 85 No. 2, pp. 185-208.

National Association of Primary Care (2019), Primary Care Home: Population Health Management, available at: https://napc.co.uk/wp-content/uploads/2019/08/NAPC-Population-heath-guide-web. pdf (accessed 16th February 2020).

NHS (2019), "Future focused finance", available at: https://www.futurefocusedfinance.nhs.uk/decisionframework-bpv (accessed 14 November 2019).

NHS Long Term Plan (2019), available at: https://www.longtermplan.nhs.uk/ (accessed 11 November 2019).

Population Health and the Population Health Management Programme at, available at: https://www. england.nhs.uk/integratedcare/building-blocks/phm/ (accessed 11 November 2019).

Ross, R.L., Sachdeva, B., Wagner, J., Ramsey, K. and Dorr, D.A. (2017), "Perceptions of risk stratification workflows in primary care health care”, Healthcare, Vol. 5 No. 4, p. 78, doi: 10. 3390/healthcare5040078.

Swanwick, T. and McKimm, J. (2011), "What is clinical leadership. . .and why is it important?", The Clinical Teacher, Vol. 8, pp. 22-26.

Symmetry Episode Risk Groups White Paper 2018, available at: https:/www.optum.com/content/dam/ optum3/optum/en/resources/white-papers/Symmetry_ERG_White_Paper_July181.pdf (accessed 18 Feburay 2020).

GP contract: Investment and Evolution (2019), "Investment and evolution: a five-year framework for GP contract reform to implement", available at: https://www.england.nhs.uk/wp-content/ uploads/2019/01/gp-contract-2019.pdf (accessed 11 November 2019).

Universal Personalised Care (2019), "Implementing the comprehensive model", available at: https:// www.england.nhs.uk/publication/universal-personalised-care-implementing-the-comprehensivemodel/ (accessed 11 November 19).

\section{Corresponding author}

Martin McShane can be contacted at: martin.mcshane@optum.com

For instructions on how to order reprints of this article, please visit our website:

www.emeraldgrouppublishing.com/licensing/reprints.htm

Or contact us for further details: permissions@emeraldinsight.com 